\title{
Theoretical evidence of the Berry-phase mechanism in anomalous Hall transport: First-principles studies of $\mathrm{CuCr}_{2} \mathrm{Se}_{4-x} \mathrm{Br}_{x}$
}

\author{
Yugui Yao, ${ }^{1}$ Yongcheng Liang, ${ }^{2,1}$ Di Xiao, ${ }^{3}$ Qian Niu, ${ }^{3}$ Shun-Qing Shen ${ }^{4}$ X. Dai, ${ }^{1,4}$ and Zhong Fang ${ }^{1,5}$ \\ ${ }^{1}$ Beijing National Laboratory for Condensed Matter Physics, Institute of Physics, Chinese Academy of Science, Beijing, 100080, China \\ ${ }^{2}$ Institute of Nano Science, Nanjing University of Aeronautics and Aetronautics, Nanjing 210016, China \\ ${ }^{3}$ Department of Physics, The University of Texas, Austin, Texas 78712-0264, USA \\ ${ }^{4}$ Department of Physics, and Center of Theoretical and Computational Physics, The University of Hong Kong, Hong Kong, China \\ ${ }^{5}$ International Center for Quantum Structure, Chinese Academy of Sciences, Beijing, 100080, China
}

(Received 27 September 2006; published 10 January 2007)

\begin{abstract}
To justify the origin of the anomalous Hall effect (AHE), it is highly desirable to have the system parameters tuned continuously. By quantitative calculations, we show that the doping-dependent sign reversal in $\mathrm{CuCr}_{2} \mathrm{Se}_{4-x} \mathrm{Br}_{x}$, observed but not understood, is nothing but direct evidence for the Berry-phase mechanism of the AHE. Systematic calculations explain well the experiment data for the whole doping range where the impurity scattering rate is changed by several orders with Br substitution. Further sign change is also predicted, which may be tested by future experiments.
\end{abstract}

DOI: 10.1103/PhysRevB.75.020401

PACS number(s): 75.47.-m, 71.20.-b, 72.15.Eb

In spite of the wide applications of the anomalous Hall effect (AHE) to characterize ferromagnetism, its origin has been a controversial subject since its discovery more than a century ago. ${ }^{1}$ The $\mathbf{k}$-space gauge fields, known as the Berry curvature, exist ubiquitously in Bloch bands where timereversal symmetry is broken, giving rise to an intrinsic AHE in ferromagnets. ${ }^{2}$ This intrinsic effect was originally derived by Karplus-Luttinger 50 years ago based on a linear response theory, ${ }^{3}$ but was disputed ever since, and until recently, extrinsic mechanisms of skew scattering and side jump were usually invoked. ${ }^{4}$ Inspired by the new understanding from the Berry phase connection, ${ }^{5-7}$ a number of quantitative studies have been successfully carried out in recent years, ${ }^{8,9}$ finding that the Berry-phase mechanism is important in various materials. However, theoretical understanding of the condition for such importance is far from clear, despite a large number of theoretical analyses based on model Hamiltonians. ${ }^{10}$ To fully explore the importance of the Berry-phase mechanism, it is highly desirable to have a systematic study of real materials in comparison to experiments when the system parameters are tuned continuously.

In this paper, we report systematical first-principles calculations on the doping dependence of the intrinsic AHE. Our material of choice is the ferromagnetic spinel $\mathrm{CuCr}_{2} \mathrm{Se}_{4}$, one of the parent compounds of a wide class of colossal magnetoresistive chalcospinels. It is well known for its high Curie temperature $\left(T_{c}=450 \mathrm{~K}\right)$ and large room-temperature magneto-optic Kerr effect, with great potential for spintronics applications. ${ }^{11}$ Experimental measurement of the AHE in this compound has been recently carried out by Lee et al., ${ }^{12}$ where they are able to tune the scattering rate by 70 -fold with $\mathrm{Br}$ substitution of Se. Our quantitative calculations explain well the experimental AHE data over the whole doping range with reasonable accuracy based on the Berry-phase mechanism. In particular, we reveal that the sharp sign change in the doping-dependent anomalous Hall conductivity, which was observed in the experiment but not discussed explicitly, is direct evidence for the Berry-phase mechanism of the AHE. The sign change is due to a large patch of high
Berry curvature in the band structure. In addition to explaining this experiment, our calculations also extend to the case of hole doping, urging further experiments on the spinel system.

The spin-polarized ground state of $\mathrm{CuCr}_{2} \mathrm{Se}_{4}$ has been calculated by the $X \alpha$ method $^{13}$ and by the linearized muffin-tin orbital method. ${ }^{11,14}$ In this work, the relativistic electronic structure is calculated self-consistently using the fullpotential linearized augmented plane-wave method with generalized gradient approximation ${ }^{15}$ (GGA) for the exchangecorrelation potential. We use the experimental lattice constant and the muffin-tin radius $R_{M T}=2.1,2.4,2.3$ bohrs for $\mathrm{Cu}, \mathrm{Cr}$, and $\mathrm{Se}$ atoms, respectively. The convergence of the present calculations has been well checked.

Figure 1 shows the calculated total and projected densities of states of the parent compound $\mathrm{CuCr}_{2} \mathrm{Se}_{4}$, where $\mathrm{Cr}$ atoms occupy the octahedral sites and $\mathrm{Cu}$ atoms occupy the tetrahedral sites. To understand the complicated electronic structure, we consider the compound as a combination of two

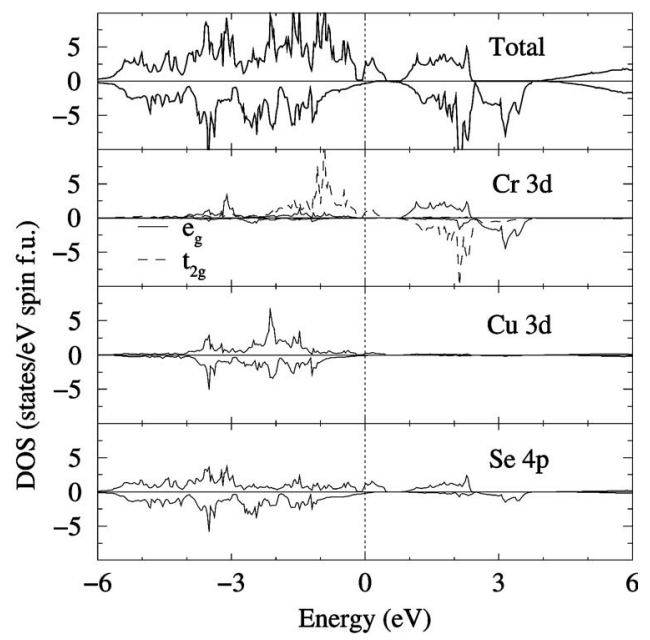

FIG. 1. The calculated total and projected densities of states of $\mathrm{CuCr}_{2} \mathrm{Se}_{4}$. The Fermi level is located at zero energy. 
TABLE I. The calculated spin, orbital, and total moments of $\mathrm{CuCr}_{2} \mathrm{Se}_{4-x} \mathrm{Br}_{x}$ in units of $\mu_{B}$.

\begin{tabular}{ccccccccc}
\hline \hline & \multicolumn{3}{c}{ Orbital moment/site } & & \multicolumn{3}{c}{ Spin moment/site } \\
\cline { 2 - 3 }$x$ & $\mathrm{Cu}$ & $\mathrm{Cr}$ & $\mathrm{Se}$ & & $\mathrm{Cu}$ & $\mathrm{Cr}$ & $\mathrm{Se}$ & Total/f.u. \\
\hline 0.0 & -0.010 & -0.0096 & -0.0030 & & -0.12 & 2.80 & -0.16 & 5.08 \\
0.2 & -0.0060 & -0.0077 & -0.0046 & & -0.11 & 2.84 & -0.15 & 5.23 \\
0.4 & -0.0017 & -0.0045 & -0.0067 & & -0.091 & 2.87 & -0.13 & 5.38 \\
0.6 & -0.0002 & -0.0027 & -0.0066 & & -0.072 & 2.91 & -0.11 & 5.61 \\
0.8 & 0.0012 & 0.0012 & -0.0063 & & -0.049 & 2.93 & -0.095 & 5.80 \\
1.0 & -0.0019 & 0.0046 & -0.0033 & & -0.022 & 2.95 & -0.072 & 5.99 \\
\hline \hline
\end{tabular}

parts: the tetrahedral $\left(\mathrm{CuSe}_{4}\right)$ clusters (in the $6+$ nominal valence), which are arranged periodically in the crystal space of the diamond structure, and the $\mathrm{Cr}$ atoms (with 3+ nominal valence) in the interstitial sites of the $\mathrm{CuSe}_{4}$ (diamond) crystal framework. As shown in Fig. 1, the electronic state of $\left(\mathrm{CuSe}_{4}\right)$ is almost non-spin-polarized (the slight polarization will be discussed later). The $\mathrm{Cu}$ is nearly in the $\mathrm{Cu}^{+}$valence state, whose $3 d$ orbitals are almost fully occupied and are away from the Fermi level. The electronic states around the Fermi level mostly come from the $\mathrm{Cr} 3 d$ and Se $4 p$ states. The $3 d$ states of $\mathrm{Cr}^{3+}$ are exchange split by about $3.0 \mathrm{eV}$, giving rise to the high spin configuration $\left(t_{2 g}^{3 \uparrow} e_{g}^{0 \uparrow} t_{2 g}^{0 \downarrow} e_{g}^{0 \downarrow}\right)$ with 3.0 $\mu_{B} / \mathrm{Cr}$ local moment. Here the $\mathrm{Cr} 3 d-\mathrm{Se} 4 p$ hybridization is an essential factor to form the final electronic structure. First, the hybridization will induce holes in the $\mathrm{Cr} t_{2 g}^{3 \uparrow}$ states, resulting in a reduced local moment and enhanced valence $\left(\mathrm{Cr}^{3+\delta}\right)$. This is evident from the slight nonoccupation of $\mathrm{Cr} t_{2 g}^{\uparrow}$ states around the Fermi level (see Fig. 1). Second, the hybridization leads to negative spin polarization of itinerant Se $4 p$ states (antiparallel to the spin moment of $\mathrm{Cr}$ ), which is crucial for the AHE in this compound. Finally, the hybridization stabilizes the ferromagnetic ground state and contributes to the high Curie temperature as discussed for $\mathrm{SrFeMoO}_{6}$ and $(\mathrm{GaMn})$ As. ${ }^{16}$ The calculated total moment is $5.1 \mu_{B} /$ f.u. for the parent compound, which is in good agreement with the experimental value of $5.2 \mu_{B}$ /f.u. ${ }^{12}$

$\mathrm{Br}$ substitution will introduce additional electrons (in addition to increasing disorder) due to the reduced negative valence of $\mathrm{Br}$ compared with Se. It is justified by the following facts that the electronic structure with doping can be described by a rigid-band shift (i.e., changing doping is equivalent to sweeping the Fermi energy) without losing the main physics for our purpose. (1) It was reported ${ }^{12}$ that $\mathrm{Br}$ substitution only affects the Curie temperature, but does not affect the ferromagnetic ground state dramatically. (2) By $25 \%$ substitution $(x=1.0)$, the lattice parameter changes only by $0.7 \% .^{17}$ (3) As a self-consistent check, the obtained electronic structures with rigid-band approximation are used to calculate the magnetic moments and give results in good agreement with experimental data. As shown in Table I, the calculated total moment per f.u. increases monotonically from $5.1 \mu_{B}$ for $x=0.0$ to $6.0 \mu_{B}$ for $x=1.0$, while the experiment shows an increase from $5.2 \mu_{B}$ to $6.0 \mu_{B} .{ }^{12}$ The calculated spin and orbital moments of each atom also agree well with the results of $\mathrm{x}$-ray magnetic circular dichroism studies. ${ }^{11}$ The orbital moment of Se sites mainly comes from its $4 p$ states due to the spin-orbit coupling.

The intrinsic anomalous Hall conductivity can be evaluated from linear response theory using the standard Kubo formula ${ }^{9}$

$$
\sigma_{x y}=\frac{e^{2}}{\hbar} \int \frac{d^{3} k}{(2 \pi)^{3}} \sum_{n} f_{n k} \boldsymbol{\Omega}_{n}^{z}(\boldsymbol{k}),
$$

with

$$
\boldsymbol{\Omega}_{n}^{z}(\boldsymbol{k})=\sum_{n^{\prime} \neq n} \frac{2 \operatorname{Im}\left\langle\psi_{n k}\left|v_{x}\right| \psi_{n^{\prime} k}\right\rangle\left\langle\psi_{n^{\prime} k}\left|v_{y}\right| \psi_{n k}\right\rangle}{\left(\omega_{n^{\prime} k}-\omega_{n k}\right)^{2}-(i \delta)^{2}},
$$

where $\left|\psi_{n k}\right\rangle$ is the eigenstate with eigenvalue $E_{n k}=\hbar \omega_{n k}, v_{x}$ and $v_{y}$ are the velocity operators, $f_{n k}$ is the Fermi-Dirac distribution function, and $\delta$ is a small parameter representing the finite lifetime broadening of the eigenstates. The $\boldsymbol{\Omega}_{n}(\boldsymbol{k})$ is a vector in $\boldsymbol{k}$ space and can be related to the Berry curvature of the Bloch state in the clean limit $(\delta=0)$-i.e., $\boldsymbol{\Omega}_{n}(\boldsymbol{k})$ $=\operatorname{Im}\left\langle\nabla_{k} u_{n k}|\times| \nabla_{k} u_{n k}\right\rangle$ with $u_{n k}$ being the periodic part of the Bloch wave function.

Figure 2 shows the calculated intrinsic $\sigma_{x y}$ as a function of the doping $x$. Let us first consider the calculated curve in the clean limit $\left(\delta=0\right.$, open circles). It is obvious that $\sigma_{x y}$ is highly nonmonotonic and changes sign twice between $x$ $=0.0$ and 0.5 ; it starts with a positive value at $x=0$, then changes sign to negative around $x=0.1$ and again to positive for $x>0.3$. The places where $\sigma_{x y}$ changes sign with varying $x$, although they appear arbitrary, are in good agreement with the experimental data (the square-cross points in Fig. 2). While a quantitative comparison with the experiment of the overall behavior of $\sigma_{x y}$ needs further analysis (as addressed below), such an agreement is a striking result, considering the fact that the calculations were done systematically for the whole doping region without adjustable parameters. In the experimental analysis, ${ }^{12}$ the sign of $\sigma_{x y}$ is dropped; only its absolute value is taken into account. Our result here, however, shows that the sign of $\sigma_{x y}$ is important and the sign change of $\sigma_{x y}$ with varying doping $x$ is a natural result of the Berry-phase mechanism of the AHE.

To make a quantitative comparison, we need to consider the effect of the finite lifetime of the eigenstates. The simplest way to do this is to assume a diagonal form of the electron self-energy and to use a single parameter $\delta$ instead 


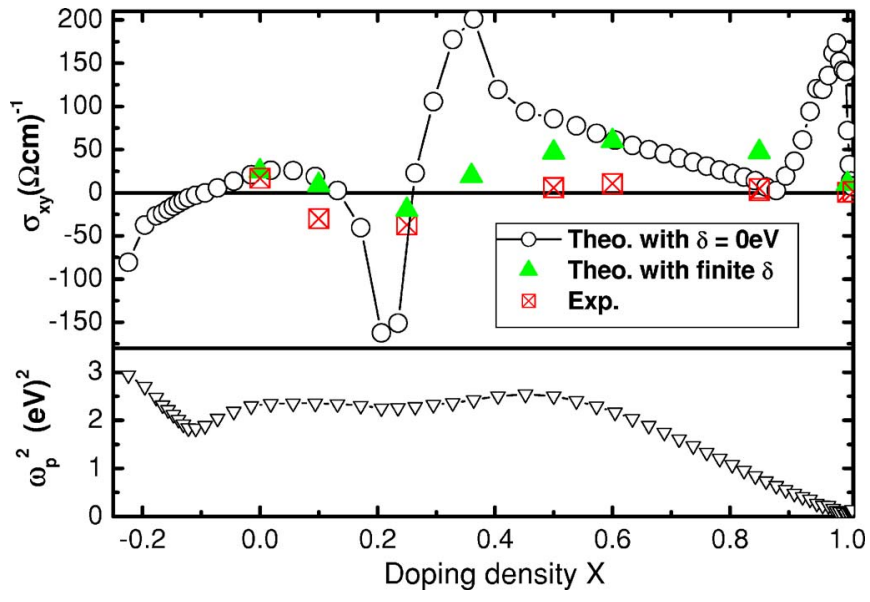

FIG. 2. (Color online) The anomalous Hall conductivity $\sigma_{x y}$ as a function of doping $x$ in $\mathrm{CuCr}_{2} \mathrm{Se}_{4-x} \mathrm{Br}_{x}$. For the theoretical results, the open circles are $\sigma_{x y}$ for the clean limit $(\delta=0)$ and the triangles are $\sigma_{x y}$ with finite $\delta$ in the Kubo formula, where the dopingdependent $\delta$ is determined from the $a b$ initio-calculated plasma frequency (shown in the lower panel) and the experimental longitudinal resistivity (see the text part for details). The square crosses are experimental results from Ref. 12.

(in the Kubo formula), thereby neglecting the vertex correction due to impurity scattering. It is worth noting that the doping-dependent $\delta$ in our approach is not adjustable parameter but instead is determined from the relaxation time $\tau=\hbar / \delta=1 /\left(\varepsilon_{0} \omega_{p}^{2} \rho\right)$, where $\rho$ is the longitudinal resistivity, adopted from Ref. 12 , and the plasma frequency $\omega_{p}$ is calculated from the band structure by

$$
\omega_{p}^{2}=\frac{e^{2}}{m^{2} \varepsilon_{0}} \sum_{n} \int \frac{d^{3} k}{(2 \pi)^{3}}\left\langle\psi_{n k}\left|p_{x}\right| \psi_{n k}\right\rangle\left\langle\psi_{n k}\left|p_{x}\right| \psi_{n k}\right\rangle \delta\left(\varepsilon_{n k}-\varepsilon_{F}\right) .
$$

The plasma frequency is actually a measurement of the ratio between the number of band carriers, $n^{*}$, and the effective mass of electrons, $m^{*}$, according to the relation $\omega_{p}^{2}=n^{*} e^{2} /\left(\varepsilon_{0} m^{*}\right)$. The triangle points in Fig. 2 are the theoretical values of $\sigma_{x y}$ after considering the effect of relaxation. It is now obvious that the calculated intrinsic $\sigma_{x y}$ is in quantitative agreement with experimental data, especially in the region around the sign change $(x=0.3)$.

The doping-dependent sign change of $\sigma_{x y}$ was observed before in other ferromagnetic alloys, such as $\mathrm{Ni}_{x-x} \mathrm{Fe}_{x}, \mathrm{Au}-\mathrm{Fe}$ and Au-Ni alloys. ${ }^{1}$ The phenomenological theory ${ }^{19}$ relates the sign change to a change of the effective spin-orbit coupling with varying chemical potential. Here our numerical calculation indicates that the sign change in $\mathrm{CuCr}_{2} \mathrm{Se}_{4-x} \mathrm{Br}_{x}$ is attributed microscopically to the topological nature of electronic bands in the Berry-phase picture. From a simplified two-band mode, it is understood that the sum of Berry curvatures over the occupied bands, $\Omega^{z}(\boldsymbol{k})=\sum_{n} f_{n k} \Omega_{n}^{z}(\boldsymbol{k})$, is spiky and the sign change occurs near the degenerate or band crossing points, which act as magnetic monopoles in momentum space. ${ }^{8}$ As a result, by summing over the Brillouin zone (BZ), $\sigma_{x y}$ is typically a nonmonotonic function of the chemical potential and exhibits sharp fluctuations. This is the case for $\mathrm{CuCr}_{2} \mathrm{Se}_{4-x} \mathrm{Br}_{x}$ as shown in Fig. 3. A similar behav-
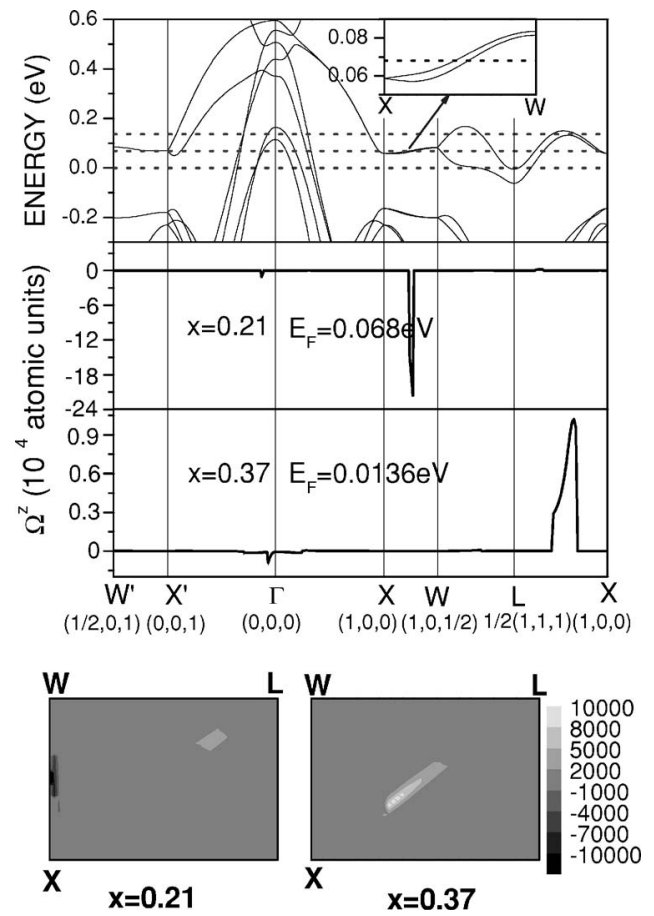

FIG. 3. The calculated band structure of $\mathrm{CuCr}_{2} \mathrm{Se}_{4-x} \mathrm{Br}_{x}$ (upper panel) and the sum of Berry curvature over the occupied bands $\Omega^{z}(\boldsymbol{k})=\Sigma_{n} f_{n k} \Omega_{n}^{z}(\boldsymbol{k})$ for two characteristic Fermi level positions corresponding to doping $x=0.21$ and 0.37 , respectively. The lower panels show the $\Omega^{z}(\boldsymbol{k})$ in a special plane of the BZ for different doping $x$.

ior was also observed in two-dimensional (2D) systems, such as the sign change in a quantum well structure. ${ }^{18}$ However, we note that the higher dimensionality in the present system makes the problem quite different. In the 3D case, the single band-crossing point cannot contribute enough weight to the sign change of $\sigma_{x y}$ due to the 3D (instead of 2D) integration of the BZ. To get enough weight, a high density of states near the band-crossing points (or near-degenerate points) are necessary (for example, the inset in Fig. 3). Due to the presence of band dispersion, it is generally hard to have all those band-crossing points occupied (or unoccupied) at each fixed chemical potential, which leads to a lower possibility for the sign change of $\sigma_{x y}$ in $3 \mathrm{D}$ than in the $2 \mathrm{D}$ case. On the other hand, $\mathrm{CuCr}_{2} \mathrm{Se}_{4-x} \mathrm{Br}_{x}$ is an isotropic 3D system where sharp sign changes of $\sigma_{x y}$ are observed. Actually, the sign changes in $\mathrm{CuCr}_{2} \mathrm{Se}_{4-x} \mathrm{Br}_{x}$ are neither from simple band crossing nor from the high-symmetric points of the BZ. As shown in Fig. 3 , the dominant negative Berry curvature for $x=0.21$ (the valley of $\sigma_{x y}$ ) and the positive Berry curvature for $x=0.37$ (the peak of $\sigma_{x y}$ ) are located at different regions of the BZ. We have tried to use an effective Luttinger Hamiltonian (fitted from our electronic structure calculations) to study the system, but the sign changes cannot be reproduced even qualitatively. This indicates that in realistic materials accurate first-principles calculations are important.

In conclusion, the doping-dependent $\mathrm{AHE}$ in $\mathrm{CuCr}_{2} \mathrm{Se}_{4-x} \mathrm{Br}_{x}$ is investigated by ab initio calculations and analyzed according to the Berry-phase picture. The good agreement between experimental and numerical results pro- 
vides strong evidence for the Berry-phase mechanism of the AHE, even when the impurity scattering rate is changed by several orders of magnitude. The disorder (extrinsic) contributions, which may also be related to the nonzero Berry curvature, ${ }^{20}$ can change the magnitude of our calculated AHE quantitatively, but they are not expected to affect such features as the sign change qualitatively. To further verify our results, we point out the following two aspects which can be justified experimentally. (i) Additional sign change is predicted from our calculation. As shown in Fig. 2, by negative doping (hole doping), we predict that $\sigma_{x y}$ changes sign from positive to negative. The hole doping can be realized experimentally by doping As instead of Br. (ii) The experimentally observed Nernst effect ${ }^{21}$ in the same compound
$\mathrm{CuCr}_{2} \mathrm{Se}_{4-x} \mathrm{Br}_{x}$ can be also checked from the present picture. 22

We acknowledge valuable discussions with Junren Shi and are grateful to Wei-Li Lee for sharing the original experimental data and for discussions. This work was supported by the Knowledge Innovation Project of the Chinese Academy of Sciences, the NSFC under Grants Nos. 10404035, 10534030, 10674163 (Y.G.Y), 90303022, 60576058, 10334090, and 10425418 (Z.F.), by the NSF under Grants Nos. DMR-0404252 and DMR-0606485 (D.X.), by the DOE under Grant No. DE-FG03-02ER45958 (Q.N.), and RGC of Hong Kong under Grant No. HKU 7042/06P (S.Q.S.).
${ }^{1}$ E. H. Hall, Philos. Mag. 10, 301 (1880); 12, 157 (1881); A. W. Smith and R. W. Sears, Phys. Rev. 34, 1466 (1929); The Hall Effect and Its Application, edited by C. L. Chien and C. R. Westgate (Plenum, New York, 1980).

${ }^{2}$ M.-C. Chang and Q. Niu, Phys. Rev. B 53, 7010 (1996); G. Sundaram and Q. Niu, ibid. 59, 14915 (1999).

${ }^{3}$ R. Karplus and J. M. Luttinger, Phys. Rev. 95, 1154 (1954); J. M. Luttinger, ibid. 112, 739 (1958).

${ }^{4}$ J. Smit, Physica (Amsterdam) 21, 877 (1955); 24, 39 (1958); L. Berger, Phys. Rev. B 2, 4559 (1970).

${ }^{5}$ T. Jungwirth, Q. Niu, and A. H. MacDonald, Phys. Rev. Lett. 88, 207208 (2002); T. Jungwirth, J. Sinova, K. Y. Wang, K. W. Edmonds, R. P. Campion, B. L. Gallagher, C. T. Foxon, Q. Niu, and A. H. MacDonald, Appl. Phys. Lett. 83, 320 (2003).

${ }^{6}$ M. Onoda and N. Nagaosa, J. Phys. Soc. Jpn. 71, 19 (2002); Phys. Rev. Lett. 90, 206601 (2003).

${ }^{7}$ F. D. M. Haldane, Phys. Rev. Lett. 93, 206602 (2004).

${ }^{8}$ Z. Fang, N. Nagaosa, K. S. Takahashi, A. Asamitsu, R. Mathieu, T. Ogasawara, H. Yamada, M. Kawasaki, Y. Tokura, and K. Terakura, Science 302, 92 (2003); R. Mathieu, A. Asamitsu, H. Yamada, K. S. Takahashi, M. Kawasaki, Z. Fang, N. Nagaosa, and Y. Tokura, Phys. Rev. Lett. 93, 016602 (2004).

${ }^{9}$ Y. G. Yao, L. Kleinman, A. H. MacDonald, J. Sinova, T. Jungwirth, D. S. Wang, E. G. Wang, and Q. Niu, Phys. Rev. Lett. 92, 037204 (2004); C. G. Zeng, Y. G. Yao, Q. Niu, and H. H. Weitering, ibid. 96, 037204 (2006).

${ }^{10}$ V. K. Dugaev, P. Bruno, M. Taillefumier, B. Canals, and C. Lacroix, Phys. Rev. B 71, 224423 (2005); N. A. Sinitsyn, Q. Niu, J. Sinova, and K. Nomura, ibid. 72, 045346 (2005); J.-I. Inoue, T.
Kato, Y. Ishikawa, H. Itoh, G. E. W. Bauer, and L. W. Molenkamp, Phys. Rev. Lett. 97, 046604 (2006); S. Onoda, N. Sugimoto, and N. Nagaosa, ibid. 97, 126602 (2006).

${ }^{11}$ A. Kimura, J. Matsuno, J. Okabayashi, A. Fujimori, T. Shishidou, E. Kulatov, and T. Kanomata, Phys. Rev. B 63, 224420 (2001); K. Ramesha and R. Seshadri, Solid State Sci. 6, 841 (2004).

${ }^{12}$ W.-L. Lee, S. Watauchi, V. L. Miller, R. J. Cava, and N. P. Ong, Science 303, 1647 (2004).

${ }^{13}$ F. Ogata, T. Hamajima, T. Kambara, and K. I. Gondaira, J. Phys. C 15, 3483 (1982).

${ }^{14}$ V. N. Antonov, V. P. Antropov, B. N. Harmon, A. N. Yaresko, and A. Y. Perlov, Phys. Rev. B 59, 14552 (1999).

${ }^{15}$ J. P. Perdew, K. Burke, and M. Ernzerhof, Phys. Rev. Lett. 77, 3865 (1996); 78, 1396(E) (1997).

${ }^{16}$ Z. Fang, K. Terakura, and J. Kanamori, Phys. Rev. B 63, 180407(R) (2001); J. Kanamori and K. Terakura, J. Phys. Soc. Jpn. 70, 1422 (2001).

${ }^{17}$ T. Kanomata, K. Shirakawa, and T. Kaneko, J. Phys. Soc. Jpn. 52, 1387 (1983).

${ }^{18}$ X. Dai, Z. Fang, Y. G. Yao, and F. C. Zhang, Phys. Rev. Lett. 96, 086802 (2006).

${ }^{19}$ P. Noziéres and C. Lewiner, J. Phys. (Paris) 34, 901 (1973).

${ }^{20}$ N. A. Sinitsyn, A. H. MacDonald, T. Jungwirth, V. K. Dugaev, and J. Sinova, cond-mat/0608682 (unpublished); S. Y. Liu, N. J. M. Horing, and X. L. Lei, Phys. Rev. B 74, 165316 (2006).

${ }^{21}$ W.-L. Lee, S. Watauchi, V. L. Miller, R. J. Cava, and N. P. Ong, Phys. Rev. Lett. 93, 226601 (2004).

${ }^{22}$ D. Xiao, Y. Yao, Z. Fang, and Q. Niu, Phys. Rev. Lett. 97, 026603 (2006). 